\title{
Evidence for maintenance of sex determinants but not of sexual stages in red yeasts, a group of early diverged basidiomycetes
}

\author{
Marco A Coelho, Paula Gonçalves and José P Sampaio*
}

\begin{abstract}
Background: The red yeasts are an early diverged group of basidiomycetes comprising sexual and asexual species. Sexuality is based on two compatible mating types and sexual identity is determined by MAT loci that encode homeodomain transcription factors, peptide pheromones and their receptors. The objective of the present study was to investigate the presence and integrity of MAT genes throughout the phylogenetic diversity of red yeasts belonging to the order Sporidiobolales.

Results: We surveyed 18 sexual heterothallic and self-fertile species and 16 asexual species. Functional pheromone receptor homologues (STE3.A1 and STE3.A2) were found in multiple isolates of most of the sexual and asexual species. For each of the two mating types, sequence comparisons with whole-genome data indicated that synteny tended to be conserved along the pheromone receptor region. For the homeodomain transcription factor, likelihood methods suggested that diversifying selection acting on the self/non-self recognition region promotes diversity in sexual species, while rapid evolution seems to be due to relaxed selection in asexual strains.

Conclusions: The majority of both sexual and asexual species of red yeasts have functional pheromone receptors and homeodomain homologues. This and the frequent existence of asexual strains within sexual species, makes the separation between sexual and asexual species imprecise. Events of loss of sexuality seem to be recent and frequent, but not uniformly distributed within the Sporidiobolales. Loss of sex could promote speciation by fostering the emergence of asexual lineages from an ancestral sexual stock, but does not seem to contribute to the generation of exclusively asexual lineages that persist for a long time.
\end{abstract}

\section{Background}

In eukaryotes sexual reproduction is an almost universal trend $[1,2]$, a fact that underlines its importance both for individual fitness and long-term survival of species [3]. Fungi in particular have evolved a rich repertoire of mating strategies and sex-determining systems that has no parallel in other eukaryotic lineages [4-6]. Sexual identity in fungi is always established in the haploid stage [7] and determined by specialized genomic regions called mating type (MAT) loci [8]. In general, fungal $M A T$ loci encode transcription factors that regulate the expression of determinants of sexual identity/compatibility, such as peptide pheromones and pheromone

\footnotetext{
* Correspondence: jss@fct.unl.pt

Centro de Recursos Microbiológicos (CREM), Departamento de Ciências da Vida, Faculdade de Ciências e Tecnologia, Universidade Nova de Lisboa, 2829-516 Caparica, Portugal
}

receptors belonging to the $\mathrm{G}$ protein-coupled receptor family [9].

In Ascomycota, and possibly also in Mucoromycotina and Chytridiomycota, the MAT loci occur in two alternate forms that determine two mating types or sexual compatibility groups [6,10-12]. This constitutes the socalled bipolar mating system. However, in Basidiomycota, two functional classes of genes can be found within MAT loci: in addition to transcriptional regulators (homeodomain transcription factors - HD1/HD2), the genes encoding pheromones and pheromone receptors $(\mathrm{P} / \mathrm{PR})$ are also located in the genomic regions that determine sexual identity. This formed the basis for the existence of a more complex mating system in some basidiomycetes. In this system two classes of MAT genes (HD1/HD2 and $\mathrm{P} / \mathrm{PR}$ ) are located in two genetically unlinked MAT loci (henceforth designated as HD and PR loci, respectively) 2829-516 Caparic 
$[9,13]$. This system is called tetrapolar, because four different mating types are generated after meiosis. Irrespective of whether the mating system is bipolar or tetrapolar, mating identity and compatibility are generally determined in basidiomycetes at two levels. First, mating and cell fusion occurs when individuals carry different alleles of genes encoding small lipopeptide pheromones and PR $[14,15]$. Following fusion of compatible partners, progression through the sexual cycle depends on a second compatibility checkpoint that relies on the formation of a heterodimeric homeodomain transcription factor (HD1/HD2) encoded also at the MAT locus $[16,17]$. The homeodomain transcription factor is only active after cell fusion because dimerization is restricted to HD1 and HD2 subunits that originate from genetically different individuals $[18,19]$. One additional relevant aspect of the tetrapolar system is that MAT loci are often highly polymorphic bringing forth species with many allelic forms that can produce up to thousands of mating types [16,20-23]. Remarkably, in basidiomycetes with many mating types (viz. mushrooms), the control of cell-cell attraction and fusion by P/PR signalling has been abandoned [21] but it is still essential for the development and maintenance of the dikaryotic state. In addition, a third MAT system, designated pseudo-bipolar, was recently found in members of the red yeast genera Rhodosporidium and Sporidiobolus, that belong to an early diverged lineage of basidiomycetes [24,25]. In both bipolar and pseudo-bipolar systems only two mating types are found but in the latter some characteristics of tetrapolar systems are also present, like the occasional occurrence of recombination within the bipolar MAT locus [24]. Among the diversity of mating systems found in fungi, various types of self-fertility (or homothallism) have also evolved [26]. In those cases, heterothallism (mating occurs only between compatible individuals carrying different $M A T$ alleles [16]), is replaced by the ability of a single clone to form sexual structures and reproduce sexually.

In addition to the various modes of sexual reproduction, asexual reproduction is also a conspicuous process of fungal propagation. This suggests that for fungi the advantages of sex, namely the promotion of adaptive evolution and the more efficient elimination of deleterious mutations [27], may in many instances be insufficient to counterbalance its disadvantages, normally associated with energy and time costs of sexuality [28]. The reasons why sexuality can be facultative in fungi seem to be related to the availability of mitotically formed spores and other modes of asexual propagation [29]. However, although the ability to reproduce mitotically in fast and efficient ways acts potentially as a strong evolutionary promoter of asexuality [30], available evidence indicates that asexuality is a derived character. Firstly, asexual species are clearly a minority (approximately $17 \%$ of all known fungal species are asexual). Secondly, asexual species very often have phylogenetically close relatives capable of sexual reproduction [31,32].

In this study we focused our attention on a basal lineage of basidiomycetes that is notorious for the co-existence of sexual and asexual species, the red yeasts of the order Sporidiobolales. This order is classified in the subphylum Pucciniomycotina, the earliest diverged lineage of basidiomycetes [33], whose most notable members are the rust fungi. A classical and presently much debated rule in fungal nomenclature imposes that asexual and sexual fungi are classified separately, in different species and genera [34]. For example, in the order Sporidiobolales sexual species are classified in the genera Rhodosporidium and Sporidiobolus whereas asexual forms are classified in Rhodotorula and Sporobolomyces [35]. Therefore, the coexistence in the same phylogenetic group of very closely related species of sexual and asexual taxa with similar ecological and physiological properties raises several questions: (i) are asexual species truly asexual having thus no traces of $M A T$ genes in their genomes? (ii) alternatively, have asexual species formed recently and therefore still exhibit (traces of) sex related genes? (iii) is mating type imbalance possibly giving rise to asexual lineages? (iv) is asexuality contributing to speciation by giving rise to new species that derive from extant sexual stocks? These questions were addressed in this study by surveying the presence and integrity of $M A T$ genes in an expanded set of red yeast species. In order to get some additional insight in the evolution of MAT loci in the Sporidiobolales, we also compared gene content and organization around MAT genes between opposite mating types and across different species.

\section{Results and Discussion}

\section{Updated phylogeny of the Sporidiobolales}

We investigated a group of 43 red yeast species representing the phylogenetic diversity in the order Sporidiobolales. These species are classified in four genera, two sexual Rhodosporidium and Sporidiobolus - and two asexual Rhodotorula and Sporobolomyces. An updated molecular phylogeny of the Sporidiobolales based on the concatenated alignment of the complete ITS region (ITS1, 5.8S and ITS2) and the D1/D2 domain of the LSU rRNA is shown in Figure 1. Three statistically well-supported clades were observed. Although such organization was somewhat anticipated in previous studies (e.g. [35,36]), the incorporation of more taxa, including species not yet formally described, and the combination of ITS and D1/D2 data, improved the resolution of the present analysis. Whereas clade A included only species of Rhodosporidium and Rhodotorula, which are characterized by the absence of forcibly discharged spores (ballistoconidia), clade $C$ contained only species of Sporidiobolus and Sporobolomyces 


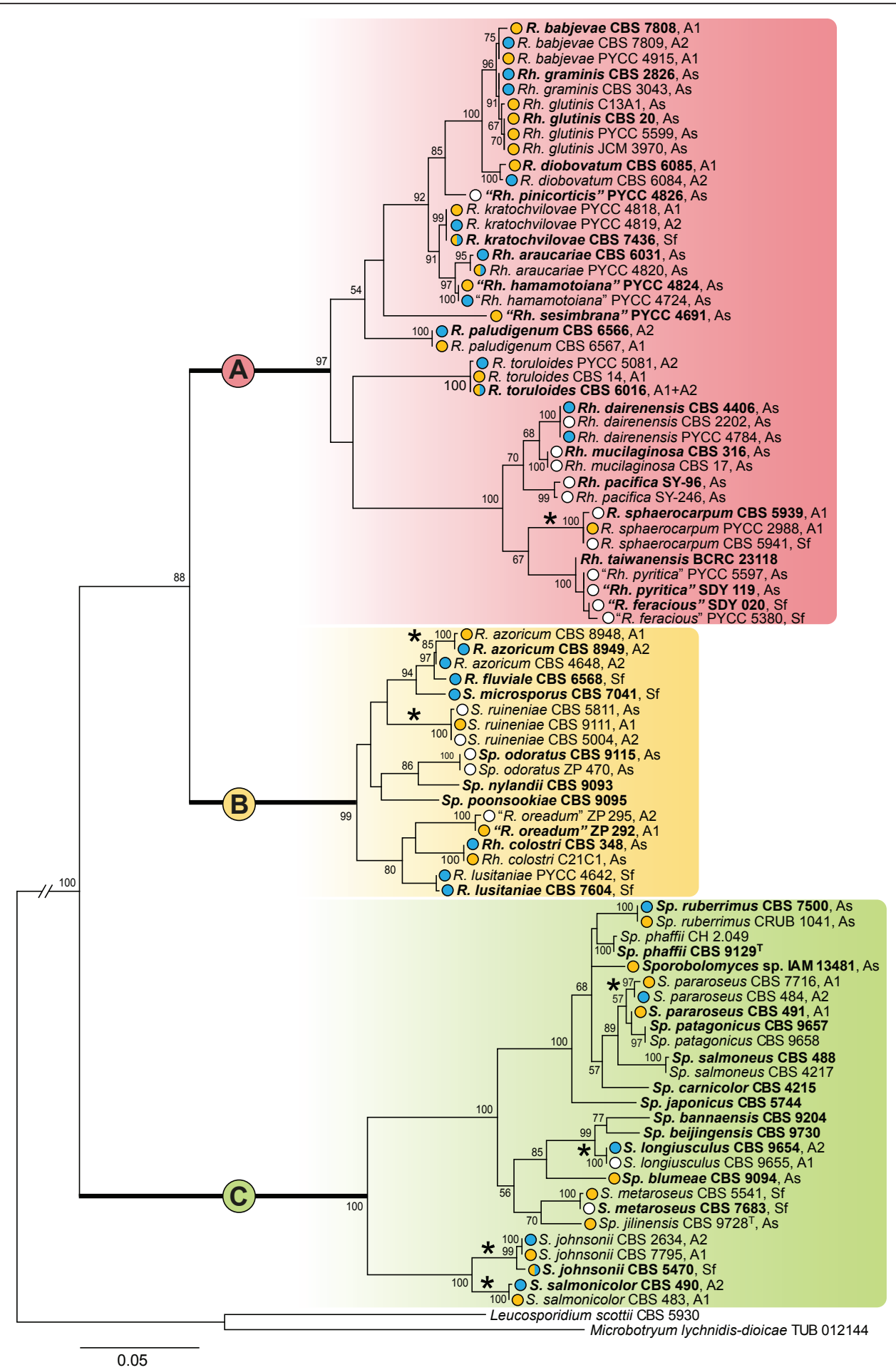

Figure 1 Phylogeny of the Sporidiobolales. Molecular phylogeny based on a concatenated alignment of the ITS region (ITS1, 5.8S and ITS2) and the D1/D2 domain of the LSU rRNA. Mating types (MAT A1 and MAT A2), asexual and self-fertile strains are designated as A1, A2, As and Sf, respectively. Circles before each strain depict the type of PR gene: yellow, STE3.A7; blue, STE3.A2; white, not detected; half-coloured circles, simultaneous detection of STE3.A1 and STE3.A2. For each species, the type strain is highlighted in boldface. A, B and C are three statistically wellsupported clades (see text for details). Abbreviations of generic names: Rhodosporidium (R.), Rhodotorula (Rh.), Sporidiobolus (S.) and Sporobolomyces (Sp.). Asterisks indicate instances where the original mating type designation was altered to match the molecular identity of the strains (see Additional File 1). Species not yet formally described are indicated by inverted commas. The tree was rooted with sequences of Microbotryum lychnidis-dioicae and Leucosporidium scottii (see methods). Branch lengths are given in number of substitutions per site. Bootstrap values (>50\%) from 1000 replicates are shown. 
that are able to form ballistoconidia. At variance with clades A and C, clade B included representatives of the four genera, which indicates that the traditional classification separating genera based on the ability to form ballistoconidia is not supported at the molecular level. It is also evident from Figure 1 that in all three clades, sexual and asexual species are completely intermingled.

\section{Pheromone receptor genes as molecular markers of mating type identity}

Multiple $H D 1 / H D 2$ alleles were found in red yeast species, and phylogenetic analyses suggested that allele diversification occurred only after each species was formed [24]. In addition, HD1/HD2 genes of different red yeast species were found to be highly divergent precluding their utilization for mating type screening purposes over a wide phylogenetic range. On the contrary, phylogenetic analyses of the PR alleles (STE3.A1 and STE3.A2) of both $M$. violaceum and $S$. salmonicolor, revealed ancient trans-specific polymorphisms $[24,37]$ as expected for genes maintained under long-term balancing selection [38]. Since only two PR alleles were present in each species examined so far, and the presence of each allele correlated in all cases with mating behaviour [24,39], PR genes can be advantageously used as markers for mating type at the molecular level across a broad range of red yeast species.

All heterothallic red yeast species within the order Sporidiobolales were originally described as having a bipolar mating behaviour. Such studies involved only standard crossing tests and strains were therefore arbitrarily assigned to either MAT A1 or MAT A2 within each species. PR genes were first identified in $R$. toruloides and were named STE3.A1 and STE3.A2 in accordance with the mating type of the strains in which they were initially characterized [39]. After the identification of the sequences of the PR genes in S. salmonicolor and S. johnsonii, it became clear that as a result of the original arbitrary assignment of mating types, each STE3 allele was associated with strains of the opposite mating type, (e.g. STE3.A1 was found in S. salmonicolor and S. johnsonii MAT A2 strains, [24]). To avoid future confusion, we previously renamed the mating type designations of these two species to match the molecular data [24]. Here we investigated at the molecular level the current mating type designations of the heterothallic species included in this work. To that end we employed a PCR-based approach with degenerate primers that specifically amplified STE3.A1 or STE3.A2 alleles. In approximately half of the heterothallic species analysed ( $R$. sphaerocarpum, $R$. azoricum, S. ruineniae, S. pararoseus and S. longiusculus) the incongruences previously observed for S. salmonicolor were also detected, and in these cases the mating type designations were altered so as to match the molecular identity of the strains (Additional File 1). It should be noted that in R. sphaerocarpum and S. ruineniae only the STE3.A1 allele could be identified (Figure 2 and Additional File 1). Nevertheless, the fact that the STE3.A1 allele was only found in strains that mated as MAT A2 suggested that the STE3.A2 allele is most probably present in MAT A1 strains, but the set of primers that we used failed to amplify it. For $R$. babjevae we investigated 25 strains (Additional File 1). Previously, mating studies had identified five strains as $M A T A 1$, four strains as MAT A2 and two strains as self-fertile. The remaining 14 strains were found to be sexually impaired and thus considered asexual. The molecular characterization of PR alleles confirmed for the sexual strains the results obtained with standard crossing tests. It also showed that one of the self-fertile strains (VKM Y-2911) contained both receptor alleles and that 11 asexual strains belonged to MAT A1 and three to MAT A2. Both mating types were present in strains isolated in Russia (six strains), Argentina (five strains) and Japan (two strains). However, the six strains isolated in Portugal belonged solely to MAT A1. Using tester strains with good mating abilities and five asexual strains for which the molecular determination of mating type had been obtained, we repeated crosses and confirmed that these strains do not mate in culture.

\section{Pheromone receptor genes in asexual species}

We subsequently investigated the presence of PR homologues (STE3.A1 and STE3.A2) in 16 asexual species classified in the genera Rhodotorula and Sporobolomyces (Figure 1 and Additional File 1). Amplification of STE3.A1 and/or STE3.A2 alleles was successful in 11 species among the 16 examined (Figure 1). When multiple strains were available for a species, both PR alleles were normally detected, signalling the presence of the two mating types in natural populations of these presumably asexual species. However, an interesting exception was found for the sibling species Rh. glutinis and Rh. graminis. Whereas all six strains of Rh. glutinis possessed the STE3.A1 allele, all four strains of $R h$. graminis exhibited the STE3.A2 allele (Figure 2 and Additional File 1). These two species are also closely related to the heterothallic species $R$. babjevae. In contrast to $R$. babjevae, only a limited number of strains of $R h$. graminis and Rh. glutinis have been isolated worldwide [40], all of which were included in the present study. For these three species, inter-species mating has been tested, but was not observed [41]. It is tempting to speculate that asexuality in $R h$. graminis and Rh. glutinis might be related to a strong but relatively recent mating type imbalance. Nevertheless, a larger sampling of both species would be required to accurately determine the mating type distribution and frequency and to elucidate if sex occurs in nature by looking for footprints of recombination, as done previously for other 


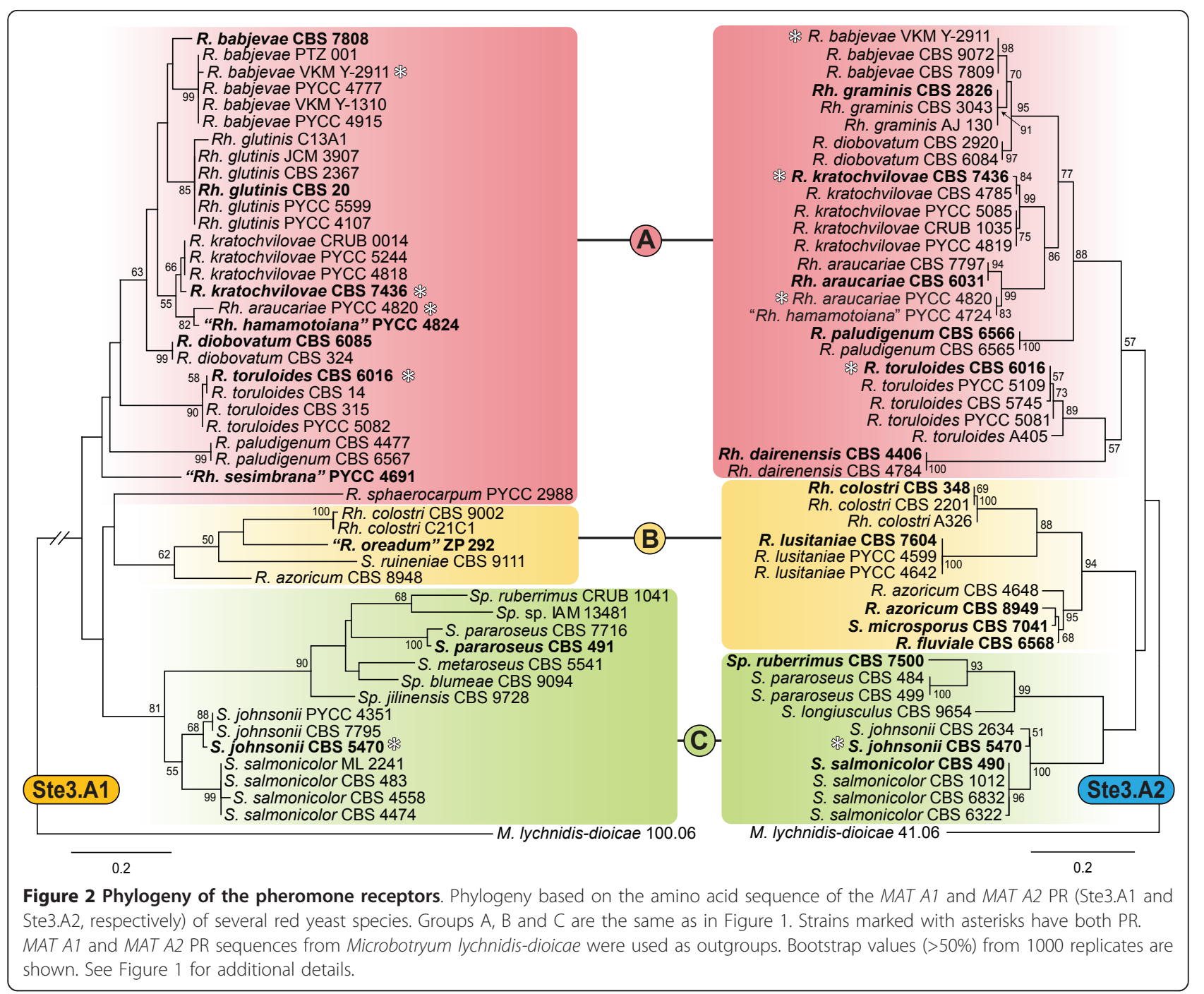

asexual fungi [42]. Moreover, it is important to note that mating type imbalance does not lead necessarily to asexuality as demonstrated in C. neoformans [43,44].

Next, we investigated the integrity of PR genes in asexual species. To this end, the complete genes were sequenced. We found that none of them showed hallmarks of degradation and that a protein sequence could be readily obtained by conceptual translation in all cases, suggesting that these genes encode functional proteins. We also inspected the PR genes in the recently released genomes of the presumptively asexual species Sporobolomyces sp. IAM 13481 (STE3.A1) and Rh. graminis WP1 (STE3.A2) (formerly designated as R. babjevae), available at the Joint Genome Institute (JGI). Again, intact and complete sequences of the PR genes were identified. Therefore, our results suggest that asexuality in red yeasts is not ancient, which is consistent with other studies that have shown that in presumed asexual ascomycetes, mating type genes are present and apparently functional $[45,46]$. It is also possible that some supposedly asexual species have retained a cryptic sexual stage that is difficult to induce in the laboratory.

In contrast to these observations, for five asexual species (Rh. mucilaginosa, Rh. pacifica, "Rh. pinicorticis", "Rh. pyritica" and Sp. odoratus) no PR alleles were found (Figure 1 and Additional File 1). With the exception of "Rh. pinicorticis" and Sp. odoratus, the remaining species are closely related to each other and belong to a lineage of clade A that includes also $R$. sphaerocarpum and $R h$. dairenensis. The phylogenetic analysis of STE3.A1 from $R$. sphaerocarpum and STE3.A2 from Rh. dairenensis, suggested that the amino acid sequences of these PR genes are unusually divergent (Figures 1 and 2). Therefore, it is conceivable that the lack of amplification in the remaining species is due to more divergent but still functional sequences. A similar explanation might apply for the amplification of $R$. sphaerocarpum STE3.A1 in only one out of six MAT A1 strains investigated. 
For $R$ h. araucariae PYCC 4820 two PR alleles (STE3.A1 and STE3.A2) were detected (Figures 1 and 2, Additional File 1). However, the nucleotide sequence of the STE3.A2 allele in this strain differed from homologous sequences of conspecific strains of the same mating type (CBS 6031 and CBS 7797; 87\% identity). Surprisingly, the STE3.A2 allele of $R h$. araucariae PYCC 4820 was found to be $100 \%$ identical to the STE3.A2 gene of "Rh. hamamotoiana" PYCC 4724. Contrary to the other strains of $R h$. araucariae, cells of strain PYCC 4820 formed a slender projection that resembled a conjugation tube, although actual conjugation between cells of the same strain or with cells of the other strains of $R h$. araucariae was not observed. It seems plausible that the phenotype of PYCC 4820 is due to the presence of $M A T$ alleles of both mating types (including the $\mathrm{P} / \mathrm{PR})$, as a result of a past hybridization event between the two species. However, incompatibilities at other loci, like for example the HD locus, are perhaps impairing progression through the sexual cycle, as no dikaryotic mycelium or teliospores were observed.

\section{Pheromone receptor genes in self-fertile species}

In homothallic species, a single strain can complete the life cycle without the need for a complementary mating partner. Although several mechanisms of homothallism have been put forward in fungi [26], the most common explanation for this sexual behaviour is that these species have both $M A T$ alleles in a single genome and can therefore form sexual structures alone. In this study we searched for the presence of both PR alleles in three species from clade $\mathrm{B}$ that contain only self-fertile strains - R. fluviale, $R$. lusitaniae and S. microsporus - but only STE3.A2 alleles were found. Although we could not rule out that the absence of amplification of STE3.A1 alleles was due to mutations in primer target sequences, we deem this explanation less likely in this case, since STE3.A1 alleles were identified and sequenced in closely related species of clade B, like $R$. azoricum and Rh. colostri. We hypothesize that for these three species self-fertility could be due to compatibility at the HD locus despite homozygosity at the PR locus. If confirmed, such a situation would parallel that found in the corn smut pathogen Ustilago maydis, in which diploid strains that are homozygous at the PR locus but heterozygous at the HD locus are able to produce teliospores that germinate and undergo meiosis in a way that is indistinguishable from that of the heterozygous diploids at both $M A T$ loci [47]. A similar self-fertile phenotype is originated in $C$. neoformans when either the $\alpha$ sex inducer gene SXI1 $\alpha$ (HD1) is introduced into $a$ cells or the $a$ sex inducer gene SXI2a (HD2) is introduced into $\alpha$ cells $[48,49]$. Nevertheless, whereas the diploid strains of $U$. maydis only produce incipient hyphae because maintenance of filamentous growth also requires heterozygosity at the PR locus [47], for the self-fertile yeast species studied we found extensive hyphal growth. Therefore, this also raises the possibility that in self-fertile red yeast species, the PR locus may be self-compatible either by constitutive activation of the pheromone receptor or by an autocrine signalling loop to produce a permanent signalling pathway. Interestingly, examples of mutations supporting both hypotheses have been reported in laboratory [50-52]. Some heterothallic species of red yeasts also include a few self-fertile strains (viz. R. kratochvilovae CBS 7436, R. babjevae VKM Y-2911 and S. johnsonii CBS 5470). In most of those cases we found that both PR alleles were present (Additional File 1) suggesting that self-fertility in red yeasts can be attained by different molecular mechanisms.

\section{Phylogenies of pheromone receptors}

Using partial sequences of STE3.A1 and STE3.A2 alleles we compared the evolutionary history of the two PR whose trans-specific phylogenies indicate that their divergence dates back to the origin of sexuality in basidiomycetes $[24,37,53]$. Phylogenies based on the protein sequences of the PR genes were constructed separately for each receptor (Ste3.A1 and Ste3.A2) in order to maximize the quality of the alignments (Figure 2). In spite of general concordance between rDNA and PR trees, three conflicts were detected in the Ste3.A1 tree. These cases correspond to the placement of $R$. diobovatum, $R$. paludigenum and $R$. sphaerocarpum outside their respective groups, by comparison with rDNA and Ste3.A2 trees (Figures 1 and 2). However, it should be noted that bootstrap values in the Ste3.A1 tree tended to be lower than those obtained for the Ste3.A2 tree and that therefore these conflicts might be due to poor resolution in the Ste3.A1 tree.

\section{Structure of the MAT A1 and MAT A2 genomic regions encompassing the alternate pheromone receptors}

We have previously observed in a restricted number of red yeast species that genomic regions around the PR locus exhibited synteny breaks, gene inversions and sequence divergence between the two mating types as a result of apparent partial suppression of recombination, while synteny was well conserved across species when $M A T$ regions of the same mating type where compared [24,39]. Here we set out to assess whether these observations also held true for the expanded set of red yeast species presently under scrutiny. Taking advantage of whole-genome data of Sporobolomyces sp. IAM 13481 (MAT A1) and Rh. graminis WP1 (MAT A2), we assessed the synteny around the PR genes in red yeast species belonging to clades $\mathrm{A}, \mathrm{B}$ and $\mathrm{C}$ (Figure 1 and Additional File 2). PCR primers anchored on the 3' end region of the genes flanking the STE3.A1 (RibL18ae and RibL6/RNAPOL) and STE3.A2 (LSm7 and RibL6/RNAPOL) yielded almost always an amplification product between 2.5 to $3.0 \mathrm{~kb}$, consistent with a gene organization identical to that found in the genomes of 
Sporobolomyces sp. IAM 13481 and Rh. graminis WP1 (Figure 3). Sequencing of the complete intervening regions confirmed the suspected synteny within the same mating type across the majority of the species (Additional File 2). However, for some species, like MAT A1 strains of $R$. paludigenum, gene order seemed to have been disrupted since no amplification products were obtained even when using different combinations of primers for the more conserved flanking genes. Another interesting exception was $S$. ruineniae CBS 5811, where a $M A T A 2$ pheromone precursor homologue similar to the RHA2.A2 of R. toruloides [39] was found between RibL18ae and RibL6 instead of the STE3.A1 gene usually present at this location (Additional File 2). This indicates that the STE3.A1 gene has been relocated to a different and presently unknown genomic location in this species.

We next examined a larger genomic region of the MAT A1 and $M A T A 2$ loci. To do so, $50 \mathrm{~Kb}$ regions encompassing the STE3.A1 gene in Sporobolomyces sp. IAM 13481 and the STE3.A2 gene in Rh. graminis WP1 were compared (Figure 3). In Rh. graminis WP1 we identified two identical putative pheromone precursor homologues (RgRHA1.A2 and $\operatorname{Rg} R H A 2 . A 2)$, similar but not syntenic to the RHA2.A2 gene of $R$. toruloides MAT A2 strains (Figure 3) [39]. The regions upstream of $\operatorname{Rg} R H A 2 . A 1$ and $\operatorname{Rg} R H A 2 . A 2$ were identical. A shorter sequence of approximately $280 \mathrm{bp}$ was also identical and was detected downstream of RNAPOL in an inverted orientation (Figure 3). This suggests that an additional copy of the pheromone precursor gene may have existed at this genomic location similarly to that found in MAT A1. Notably, MAT A1 pheromone genes in $S$. salmonicolor and $R$. toruloides were found to be embedded in conserved inverted repeats [39], a situation earlier documented in the basidiomycete human pathogen C. neoformans [54]. These repeats are likely maintained by intra-allelic gene conversion to ensure maintenance of gene function in face of the absence of meiotic recombination characteristic of these regions $[55,56]$.

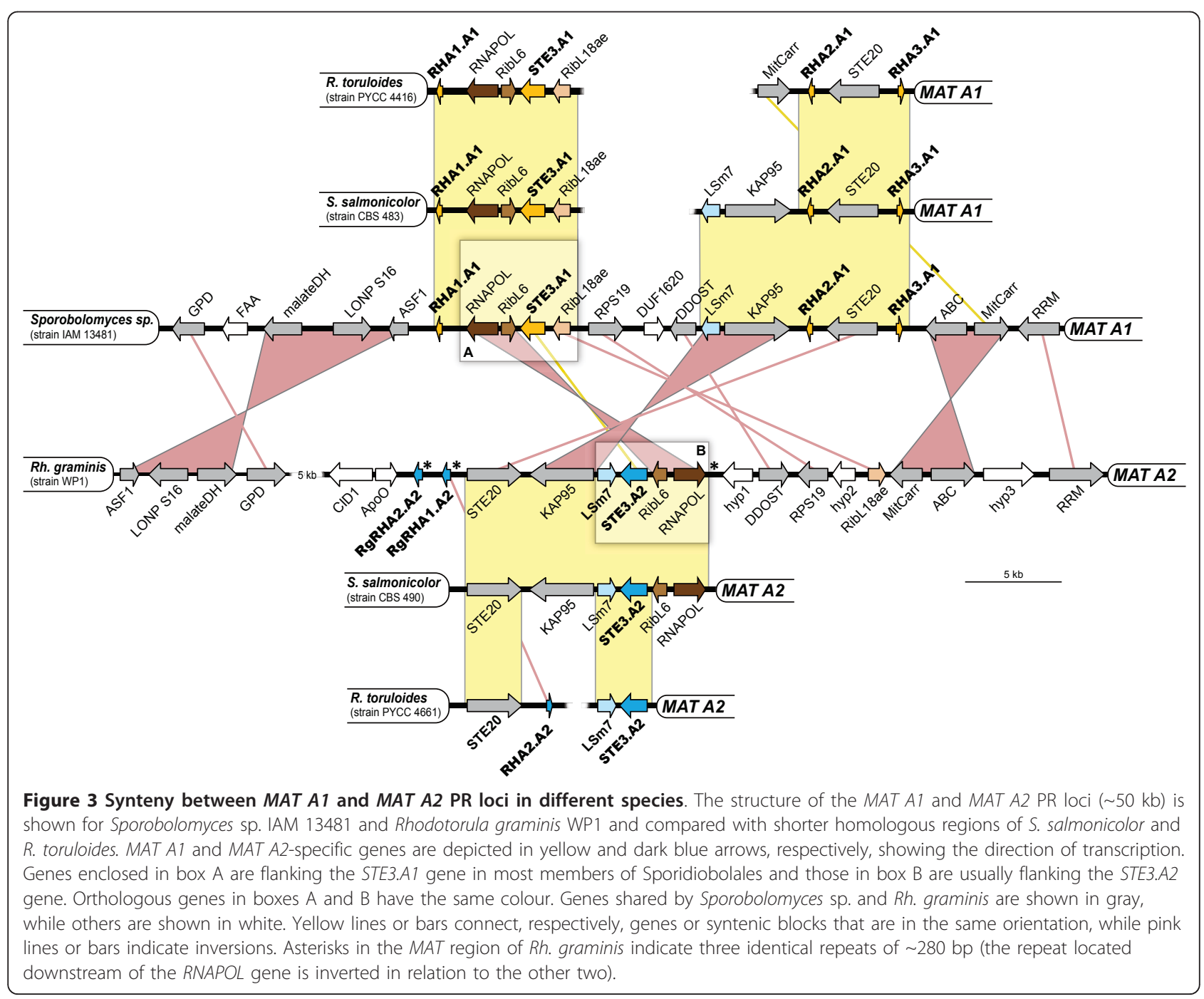


Our comparison of the gene content of the MAT genomic regions shows that gene content is similar despite the phylogenetic distance between Sporobolomyces sp. and Rh. graminis (Figures 1 and 3 ) and that apparently functional copies of each allele have been retained in both MAT loci. This is in contrast to genes encoded in sex chromosomes of diploid organisms, for which degeneration and loss of one functional copy frequently occurs [57]. Maintenance of both alleles in fungal sex-determining regions, including the $M A T$ loci of red yeasts, is most probably related to the fact that fungi commonly occur as haploids in the environment, and thus gene degeneration or loss is very likely to be detrimental. However, gene order seems to have been deeply altered during mating type divergence and many gene blocks were found in inverted positions, probably restraining recombination between opposite MAT regions (Figure 3). On the contrary, within the same mating type, the analysed regions were found to be highly syntenic even between more distantly related species (Figure 3 ).

\section{Diversity and evolution of homeodomain transcription factors}

We have previously observed that the $H D 1 / H D 2$ alleles of some red yeasts are much more divergent than the correspondent PR genes [24], and therefore less suitable for comparisons involving more distantly related species. Nevertheless, we aimed to ascertain if the genes encoding these transcriptional regulatory factors appear to be functional within a more expanded group of red yeasts including sexual and asexual strains and species and to evaluate their pattern of evolution. To this end, partial sequences of $H D 1 / H D 2$ alleles were obtained for six species and their phylogeny is shown in Figure 4. In clade A, formed by S. johnsonii and S. salmonicolor, the topology of the HD1/ HD2 tree conflicts with the rDNA (Figure 1) and Ste3 (Figure 2) trees because $S$. johnsonii CBS 2634 harbours an HD1/HD2 S. salmonicolor-type allele and the converse situation occurs for S. salmonicolor strains CBS 1012, CBS 4474 and IHMT 2446/96 (Figure 4a). Such discrepancies suggest that the two species are not fully genetically isolated and that mating with exchange of $H D 1 / H D 2$ alleles is still possible. In clade $B$, the lack of strict species-specificity of $H D 1 / H D 2$ alleles is also evident for the complex R. babjevae - Rh. glutinis - Rh. graminis. Our observation that pheromone precursor genes seem to encode very similar or even identical peptides in closely related species, possibly precluding discrimination between species prior to cell fusion is also in line with this. For example, only synonymous substitutions were detected when analyzing the RHA2.A1 genes encoding the tandem pheromone peptides from different strains of $S$. salmonicolor and S. johnsonii (Additional File 3). This results in the production of identical peptide pheromones, a circumstance that is likely to facilitate interspecies mating.

Judging from available data, a geographic structure for $H D 1 / H D 2$ allele distribution seems to be lacking since strains of $R h$. glutinis from different regions have the same $H D 1 / H D 2$ alleles and in $R h$. graminis different alleles were recovered from the same region (Figure 4a and Additional File 1). For most of the species studied each strain had a unique $H D 1 / H D 2$ allele although in Rh. glutinis four strains shared the same $H D 1 / H D 2$ allele. For two strains of $R$. babjevae (VKM Y-1310 and CBS 9072) it seems that a recent recombination event between the two MAT regions gave rise to strains with different $P R$ alleles that share the same HD1/HD2 allele, consistent with the proposed pseudo-bipolar system [24] (Figure 4a). Although the phylogenies and diversity of $H D 1 / H D 2$ alleles in S. salmonicolor and S. johnsonii suggested that such events were likely to occur albeit at low frequency, they had been thus far detected only in laboratory crosses [24].

Allele divergence as inferred by branch length seems to be lower for the species in clade A, solely composed of sexually competent strains, than in clade B, which is dominated by asexual strains (Figure 4a). Adaptive changes in protein-coding genes may be detected by comparing the number of synonymous $\left(\mathrm{d}_{\mathrm{S}}\right)$ and nonsynonymous $\left(\mathrm{d}_{\mathrm{N}}\right)$ substitutions per site, with the ratio $\omega=\mathrm{d}_{\mathrm{N}} / \mathrm{d}_{\mathrm{S}}$ providing a measure of selection at the protein level. We used seven codon-site models of variable ratios of $\omega$ across site, as implemented in PAML version 4.0 [58], to investigate the evolutionary constraints acting on the $\mathrm{N}$-terminal domain of HD1 alleles, which is known to define mating specificity, as well as the initial part of the homeodomain motif. This analysis was done separately for each clade, and for clade B it involved sexual and asexual species and sexual and asexual strains within a species ( $R$. babjevae), whereas clade A was composed solely of sexual strains (Figure 4). Detailed descriptions of the models, likelihood ratio statistics and parameter estimates for each dataset are given in Additional File 4. $R$. diobovatum was excluded because it was too divergent from the remaining species in clade B, presenting $\mathrm{d}_{\mathrm{S}}>2.0$ in most pairwise comparisons. We found that the $H D 1$ gene evolves faster in clade A than in clade $B$ given the averaged values of $\omega$ for the two clades (0.504 vs. 0.172 , respectively) obtained from model M0 (one ratio), which assumes a single $\omega$ for all codons in the sequence. However, synonymous substitution rates were significantly higher in clade $\mathrm{B}$ than in clade $\mathrm{A}(P<0.001$, Mann-Whitney U-test), indicating that in clade $B$, the $H D 1$ gene may be under fewer functional constraints, possibly due to a lower strength of codon usage bias associated with changes in the level of gene expression [59]. For the dataset of clade A, models M2a (selection) and M8 (beta\&()), which allow for positive selection at a subset of 


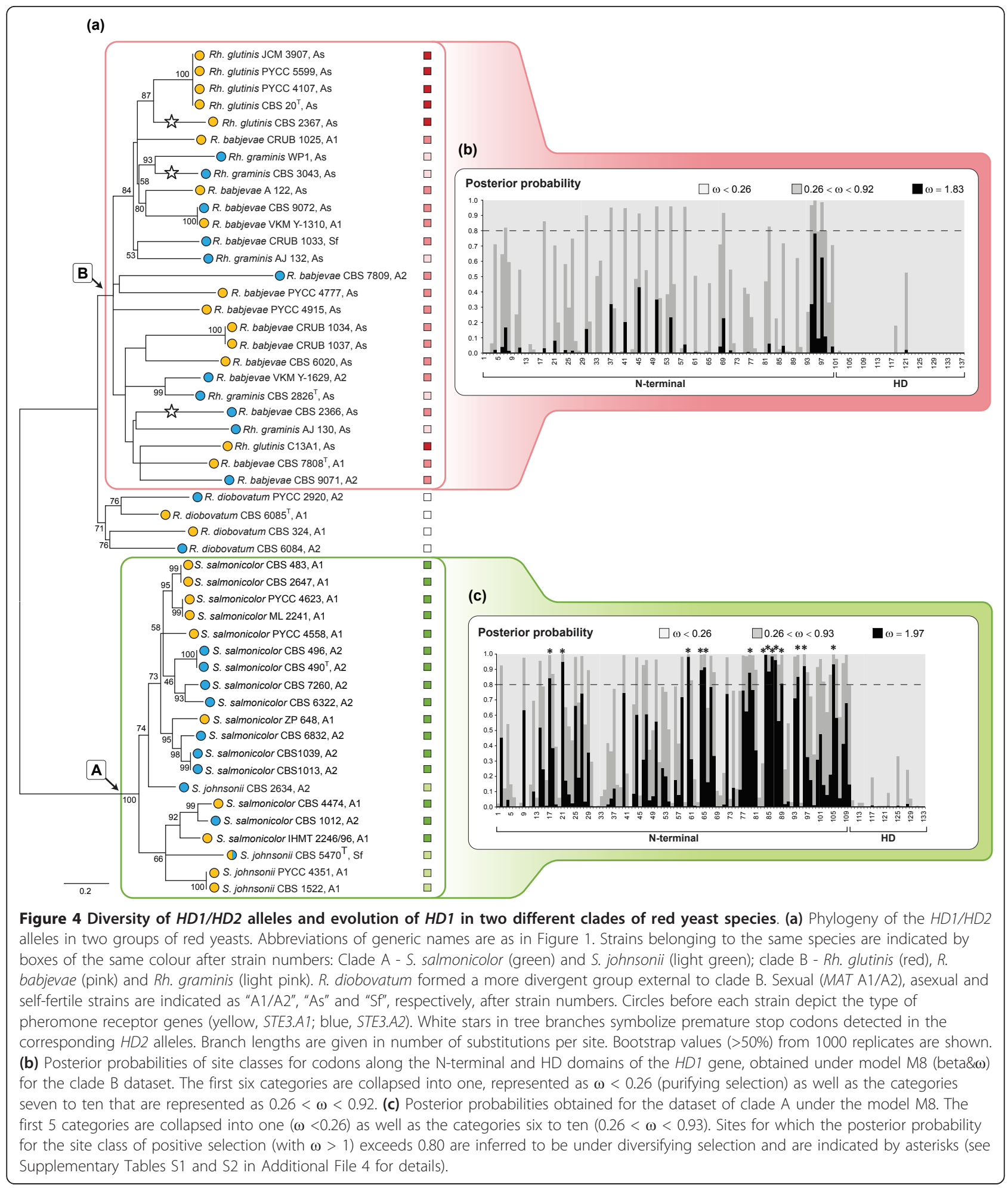

sites, fitted the data better than the null models M1a (nearly-neutral), M7 (beta) and M8a (beta\& $\omega_{\mathrm{s}}=1$ ), which do not allow for positive selection (Supplementary Tables S1 and S2 in Additional File 4). Parameter estimates under model M8 (beta\& $\omega$ ) for the dataset of clade A suggest that $\sim 22 \%$ of the sites are under diversifying selection $(\omega>1)$ with $\omega_{\mathrm{s}}=1.97$. These sites were found within the regions determining $M A T$ specificity, in accordance with previous observations [24]. Nevertheless, the average $\omega$ along the $\mathrm{N}$-terminal region was $<1$, as also observed in other 
systems such as the $S$-loci of plants [60,61]. Additionally, sites within the homeodomain motif exhibited low $\omega(\omega<$ 0.26 ) indicative of strong purifying selection. The posterior probabilities obtained under model M8 for the different site classes (see Supplementary Table S1 in Additional File 4) are shown in Figure 4. For the clade B dataset (R. babjevae, Rh. glutinis and Rh. graminis), positive selection models did not fit the data better than the models implying neutral evolution indicating that positive selection is not driving the evolution of the HD1 gene in this clade (see Supplementary Table S2 in Additional File 4). Moreover, model M8a (beta\& $\omega_{\mathrm{s}}=1$ ) where an extra site class was constrained to have $\omega=1$, seems to fit the data better than other models (Supplementary Table S2 in Additional File 4) consistent with the identification of relaxed purifying selection [62]. This may be a consequence of loss of sexual competence in Rh. glutinis, $R h$. graminis, and in several strains of $R$. babjevae. In line with this, stop codons were found in N-terminal regions of the HD2 gene of Rh. graminis CBS 3043 and R. babjevae CBS 2366, which is asexual. In Rh. glutinis CBS 2367 a dinucleotide repeat $(\mathrm{CT})_{13}$ insertion followed by a premature stop codon most likely renders this gene non-functional. Although C-terminal truncations of other HD proteins, including Sxila in C. neoformans [44], did not affect their functionality, the early stop codons found in asexual strains of red yeasts are located in the $\mathrm{N}$-terminal region of the protein, thus presumably originating very short (non-functional) proteins devoid of homeodomain motifs. For the $H D 1$ gene, no stop codons were found in the analysed regions and the evolution of the homeodomain motif seems to be dominated by purifying selection in species of both clades. While this may suggest that HD1 proteins have additional roles that do not require heterodimerization (a possibility so far poorly explored in basidiomycetes), another possibility is that this gene is losing its mating specific role in species of clade $B$ as a consequence of relaxed functional constraints. Therefore, it seems possible that the sequence divergence of the $H D 1 / H D 2$ genes examined here is governed both by diversifying and by relaxing selection, and that this may be either the cause or the consequence of loss of sexual competence by individual strains.

Our results suggest that loss of sex might not occur uniformly across the Sporidiobolales. Different molecular evolution patterns may be related with distinct selective constraints acting on different species. S. salmonicolor and S. johnsonii exemplify a case of strictly sexual red yeasts whose $H D 1 / H D 2$ alleles exhibit low $\mathrm{d}_{\mathrm{S}}$ values possibly due to some form of constraint on synonymous substitutions like codon bias [63,64] (Figures 4), but where allele specific regions are evolving under positive selection. The study of maintenance or loss of sex in red yeast could benefit from a larger population sampling that would allow to look for genetic footprints of recombination [42]. Nevertheless, even fungi shown to be purely clonal from a population genetic perspective (viz. Penicillum marneffei), also present $M A T$ genes in their genomes [65], suggesting that sex has been lost only recently.

\section{Conclusions}

We examined 216 strains belonging to 32 species of the Sporidiobolales and found apparently functional PR homologues for most strains, irrespective of their sexual or asexual status. In addition, we observed that all asexual species have phylogenetic close relatives that mate well in laboratory conditions and we think it is likely that asexual red yeasts have lost the ability to cross, although this has not (yet) resulted in the degeneration or loss of MAT genes in most cases. However, we detected mutations likely driving loss of function in HD2 homologues in some asexual strains here investigated, which seems to be consistent with relaxed selection as a consequence of loss of sex.

Taken together, our results suggest a fuzzy separation between sexual and asexual red yeast species, illustrated by the existence of asexual strains within sexual species and by the conservation of MAT regions in asexual species. The co-existence of sexual and asexual reproduction in red yeasts probably facilitates the emergence of asexual lineages. For example, Rh. glutinis and Rh. graminis seem to derive from populations of a heterothallic $R$. babjevaelike ancestor that have independently lost the ability to mate. Notably, sexual reproduction in the latter species seems to be lost easily, as many of the $R$. babjevae strains we investigated were unable to mate.

Several authors have argued that most asexual fungi are evolutionary dead ends awaiting extinction $[29,66,67]$. This fits well with our observations in red yeasts since we did not find evidence among extant species for ancient events of loss of sexuality. Instead, we uncovered abundant substantiation for frequent and recent such events, leading in some cases to the isolation of lineages and eventually to speciation but never to the generation of exclusively asexual phylogenetic lineages that persist for a long time.

\section{Methods}

\section{Strains and mating tests}

The list of strains studied and relevant information pertaining to them is given in Additional File 1. To study sexual compatibility, pairs of 2-4 day-old cultures were mixed on corn meal agar (Difco), incubated at $18{ }^{\circ} \mathrm{C}$ $22{ }^{\circ} \mathrm{C}$ for 1 week and examined microscopically using phase-contrast optics for production of mycelium with clamp connections and teliospores (globose and thickwalled resting structures that are the site of karyogamy). 
PCR detection and sequencing of MAT A1 and MAT A2 pheromone receptor genes and the conserved surrounding regions

Diagnostic PCRs with degenerate primers for STE3.A1 and STE3.A2 alleles were carried out to directly identify the PR genes and (re)assign the molecular mating types in most of the studied red yeast strains (Additional Files 1 and 5). For some selected strains the amplification products were purified and sequenced with the same primers (Additional File 1). However, this approach failed to yield amplification products for some of the species. In these cases, STE3.A1 and STE3.A2 alleles were detected by long range PCR using primers based on the available sequences of the more conserved genes flanking the STE3.A1 in Sporobolomyces sp. IAM 13481 (genes RibL18ae and RibL6 or RNAPOL) and STE3.A2 in Rh. graminis WP1 (genes $L S m 7$ and RibL6 or RNAPOL) (Additional File 5). The amplification products were subsequently sequenced by primer-walking (Additional File 5). This latter approach was also used to confirm if synteny in the immediate vicinity of the PR genes in MAT A1 and MAT A2 strains was maintained across an expanded set of red yeast species (Additional Files 2 and 5). For each yeast species, PCR reactions, cycling conditions and primer sequences are specified in Additional File 5.

\section{PCR amplification and sequencing of HD1/HD2 region and the RHA2 pheromone precursor gene}

Using the HD1/HD2 sequences of $S$. salmonicolor and Sporobolomyces sp. IAM 13481 [24], a Blastn search was performed in the NCBI Trace Archive database of Rh. graminis WP1 and the sequences corresponding to positive hits were assembled. The genome of Rh. graminis WP1 http://genome.jgi-psf.org/Rhoba1_1/Rhoba1_1.home.html was employed to confirm the assembled region, which is located in scaffold 16 (base positions 15900-16300). The deduced HD1 and HD2 protein sequences of Rh. graminis, S. salmonicolor and Sporobolomyces sp. IAM 13481 were aligned and the conserved regions were used to design degenerate primers (MC118 and MC120) to amplify and sequence the corresponding $\mathrm{N}$-terminal and intergenic regions of the $H D 1 / H D 2$ genes in several strains of $R$. babjevae, Rh. graminis, Rh. glutinis and $R$. diobovatum (Additional Files 1 and 5). GenBank accession numbers are listed in Additional File 1. The RHA2 gene was amplified in selected S. salmonicolor strains using primers (MC040 and MC073) that anchored on the STE20 and KAP95 flanking genes. The amplification products were subsequently sequenced by primer-walking. For PCR reactions, cycling conditions, primer sequences and GenBank accession numbers see Additional File 5.

\section{Sequence data and phylogenetic analyses}

For the species tree, PCR amplification and sequencing was done as previously described [41]. Sequences of both regions were concatenated and subsequently aligned using ClustalW 1.4 [68] included in the BioEdit software [69]. The phylogenetic tree was inferred by Maximum Likelihood (ML) with PhyML [70]. FindModel, a web implementation of ModelTest [70,71], was used with the Akaike information criterion (AIC) to select the model that best fit our data. The General Time Reversible model with a discrete gamma distribution was chosen $(\mathrm{GTR}+\mathrm{G}$; shape parameter $=0.1947)$. Microbotryum lychnidis-dioicae (DQ366868/AY877416) and Leucosporidium scottii (AF0700419/AF444495) were used to root the tree. For the HD1/HD2 tree (Figure 4), nucleotide sequences were aligned with MUSCLE [72] since it produced a better alignment for such highly divergent sequences. The phylogenetic tree was also inferred by ML based on the TN93+G+I model (shape $=2.0446$; pinv $=0.0524)$. Protein sequences of the PR (Ste3A1 and Ste3A2) were deduced from the DNA sequences after removal of putative introns, either manually or using AUGUSTUS software [73]. ProtTest [74] with Akaike information criterion (AIC) was used to select the model that best fit our data and phylogenetic trees were inferred with maximum likelihood (ML). The MtREV+G+F model (shape parameter $=1.198)$ [75-77] was chosen for the Ste3A1 tree and the $\mathrm{WAG}+\mathrm{G}+\mathrm{F}$ (shape parameter = 1.0496) [76-78] was selected for the Ste3A2 dataset. Microbotryum lycnhidis-diocae pr-MatA1 (EF584742) and pr-MatA2 (ABU62846) were used to root Figure 2 trees. Bootstrap values were calculated from 1000 replicates for all trees. GenBank accession numbers of the novel sequences are listed in Additional File 1 and raw phylogenetic data (alignments and tree files) is included in Additional File 6.

\section{Estimation of the evolution rates of $5^{\prime}$ end and homeodomain regions of the $H D 1$ gene}

The region of the HD1 gene corresponding to the 5'end and the initial part of the homeodomain motif was amplified and sequenced for the strains indicated in Additional File 1, which lists also GenBank accession numbers. The deduced protein sequences were first aligned with MUSCLE [72] and then poorly aligned regions and regions containing indels were removed. The resulting alignment was used to obtain the corresponding nucleotide (codonbased) alignment. Likelihood-based tests were used to investigate the type of evolutionary pressure acting on the sequenced region of the HD1 gene, using the CODEML program within PAML software (version 4.4) [58]. The analyses were carried out using two separate datasets corresponding to clade A (S. salmonicolor and S. johnsonii, sexual) and clade B (Rh. glutinis and Rh. graminis, asexual; $R$. babjevae and $R$. diobovatum, sexual but several strains of the former are asexual). Synonymous substitution rates $\left(d_{S}\right)$ were estimated for each dataset by comparing rates 
between taxa in all possible combinations (runmode $=-2$ in PAML). Since $d_{S}$ values obtained from $R$. diobovatum were $>2.0$ in several pairwise comparisons, strains of this species were removed from subsequent analyses. MannWhitney U-test was used to examine whether or not the $\mathrm{d}_{\mathrm{S}}$ pattern differed between both clades. The tree files used as an input file for CODEML were produced by Maximum Likelihood (ML) with PhyML [70] and using FindModel with AIC to select the model that best fit each dataset $[\mathrm{T} 92+\mathrm{G}$ (shape parameter $=1,085)$ for clade $\mathrm{A}$ and TN93+G+I (shape $=1.135$; pinv $=0.164)$ for clade B] .

Codon-based likelihood analysis was conducted under seven site models $[79,80]$ implemented in CODEML, and their main characteristics are described in Supplementary Methods (Additional File 4). To verify which models fit the data better, likelihood ratio tests (LRTs) were performed by comparing twice the log-likelihood difference ( -2 two nested models using $\Delta l$ ) between two nested models using a $\chi^{2}$ distribution, with the number of degrees of freedom (df) equal to the difference in the number of parameters between models [81]. To examine whether or not the analysed region evolves under positive selection, models that allow a class of codons with positively selected sites (i.e. $d_{N} / d_{S}$ or $\omega>1$ in models M2a and M8) were compared with their nested neutral models (M1 and M7, respectively), using $2 \mathrm{df}$. [81,82]. In addition, comparison between models M8 and M8a (1 df) also allowed testing for evidence of positive selection and to eliminate the potential identification of relaxed purifying selection [80] (see Supplementary Methods in Additional File 4). According to the LRTs, when the positive selection models fitted the data significantly better that the neutral models, the identification of sites evolving under positive selection was obtained by Bayes empirical Bayes (BEB) calculation of posterior probabilities (pp) for site class implemented in models M2a and M8 [83]. Codon sites with $\omega>1$ and $\omega<0.25$ and pp values $>80 \%$ were considered to be under positive or purifying selection, respectively. Likelihood ratio statistics and parameter estimates for the two datasets are listed in Supplementary Tables S1 and S2 (Additional File 4).

\section{Additional material}

Additional file 1: List of species/strains used in this study and relevant information pertaining to them. GenBank accessions numbers are given for each genomic region analysed in this study. Sequences retrieved from the CBS culture collection database ('CBS seq.') or from genome projects ('JGl genome') are indicated. Mating behaviour as originally determined by classical mating tests ('old') and as reassigned in this study based on the molecular data ('new') is indicated when needed: $\mathrm{A} 1, \mathrm{~A} 2$, As and Sf stand for mating type A1, A2, asexual and selffertile strains, respectively. The "molecular mating type" as identified by PCR detection of the pheromone receptor alleles STE3.A1 and STE3.A2 is depicted by yellow and blue circles, respectively. White circles indicate strains for which PCR detection gave negative results and 'n.d.' stands for 'not determined'. Strains highlighted in boldface were used in Figure 1 and those indicated by thick-lined blue or yellow circles were used in Figure 3. Sequences accession number of the HD1/HD2 alleles used in Figures $4 \mathrm{~b}$ and $4 \mathrm{c}$ are also highlighted in boldface. Abbreviations: Rhodosporidium (R.), Rhodotorula (Rh.), Sporidiobolus (S.), Sporobolomyces (Sp.), Type strain (T), Lectotype (LT), Authentic strain (AUT). Species not yet formally described are shadowed in light yellow and those for which no molecular mating type was determined are shadowed in gray.

Additional file 2: Synteny of the genomic regions flanking the alternate PR in MAT A1 and MAT A2 strains of several red yeasts species. (a) Simplified illustration of the tree represented in Figure 1, indicating the phylogenetic placement of the species where gene organization in the vicinity of the pheromone receptor genes was determined. Clades A, B and C are the same as in Figure 1 and 2. (b) The mating behaviour (A1, A2, As and Sf stands for mating type A1, A2, asexual and self-fertile, respectively), the molecular mating type (STE3.A7, yellow circles; STE3.A2, dark blue circles; STE3.A1 and STE3.A2, halfcoloured circles) and the obtained genomic regions flanking the pheromone receptor alleles (STE3.A1 and STE3.A2) are shown for each strain. Orthologues are shown in the same colour. In Rhodosporidium lusitaniae, the intervening region between the STE3.A2 and the RibL6 genes (faint line) was not sequenced. Abbreviations of generic names are as in Figure 1 and the remaining features are represented as in Figure 3.

Additional file 3: Pheromone precursors of different red yeast species. (a) The organization of the genomic region encompassing the MAT Al pheromone precursor genes in Sporobolomyces sp. IAM 13481 is shown on top. Coding regions of the pheromone precursor genes (RHAT, RHA2 and RHA3) are depicted by gray arrows, indicating the direction of transcription. (b) Alignment of the Rha2 pheromone precursor of different MAT A1 red yeast species/strains (Ss, Sporidiobolus salmonicolor; Sj, Sporidiobolus johnsonii; Sp, Sporobolomyces sp. IAM 13481; Rt, Rhodosporidium toruloides). Amino acids differing from the $S$. salmonicolor strain CBS 483 are shown in red. Sequence repeats proposed to represent the peptide moiety of the mature pheromone are shadowed and those resembling the CAAX motif are underlined. (c) Phylogenetic tree showing the relationships between RHA2 genes from the indicated red yeast species, based on the alignment of their coding sequences. Groups are the same as in (b). Sequences of strains depicted in boldface are shown in (b). The tree was inferred using Maximum Parsimony. Bootstrap values from 1000 replicates are shown in the tree nodes.

Additional file 4: Supplementary Table S1. Likelihood ratio statistics and parameter estimates for the dataset of clade A (S. salmonicolor and S. johnsonii) as inferred under seven models of $\omega$ over codons. Supplementary Table S2. Likelihood ratio statistics and parameter estimates for the dataset of clade B (R. babjevae, Rh. glutinis and Rh. graminis) as inferred under seven models of $\omega$ over codons. Supplementary Methods. Model characteristics and parameters for CODEML ctl file.

Additional file 5: List of primers and specific PCR conditions used to amplify the indicated regions.

Additional file 6: Supplementary Files 1. Files used to construct the phylogenetic tree represented in Figure 1 (alignment file: "Fig1Align.fas"; Tree files: "Fig1TreeNewick.nwk ", "Fig1TreeNexus.nex", and

"Fig1TreeRootNexus.nex"). Supplementary Files 2. Files used to construct the phylogenetic trees represented in Figure 2 (alignment file for Ste3a1: "Fig2A1Align.fas"; Tree files: "Fig2A1TreeNewick.nwk", "Fig2A1TreeNexus. nex", "Fig2A1TreeRootNexus.nex"; alignment file for Ste3a2: "Fig2A2Align. fas"; Tree files: "Fig2A2TreeNewick.nwk", "Fig2A2TreeNexus.nex",

"Fig2A2TreeRootNexus.nex"). Supplementary Files 3. Files used to construct the phylogenetic tree represented in Figure 4 (alignment file: "Fig4Align.fas"; Tree files: "Fig4TreeNewick.nwk", "Fig4TreeRootNexus.nex"). Nexus files can be viewed by Mesquite software http://mesquiteproject. org/mesquite/download/download.html).

\section{Acknowledgements}

We thank V. Robert, M. Groenewald (CBS, The Netherlands) and C.P. Kurtzman (NRRL, USA) for providing yeast strains, and A. Phillips for critical 
reading of the manuscript. MAC holds a grant (SFRH/BD/29580/2006) from Fundação para a Ciência e a Tecnologia, Portugal. This work was supported by grant PTDC/BIA-GEN/112799/2009 from Fundação para a Ciência e a Tecnologia, Portugal. The funder had no role in study design, data collection and analysis, decision to publish, or preparation of the manuscript. We acknowledge access to the genome sequences of Sporobolomyces sp. strain IAM 13481 and Rhodotorula graminis WP1 (sequence data was produced by the US Department of Energy Joint Genome Institute http://www.jgi.doe. gov/ in collaboration with the user community).

\section{Authors' contributions}

MAC, PG and JPS conceived the study and designed the experiments. MAC performed the experiments. MAC, PG and JPS analyzed and interpreted the data and wrote the paper. All authors have read and approved the final manuscript.

Received: 13 April 2011 Accepted: 31 August 2011

Published: 31 August 2011

\section{References}

1. Dacks J, Roger AJ: The first sexual lineage and the relevance of facultative sex. J Mol Evol 1999, 48:779-783.

2. Ramesh MA, Malik SB, Logsdon JM Jr: A phylogenomic inventory of meiotic genes; evidence for sex in Giardia and an early eukaryotic origin of meiosis. Curr Biol 2005, 15:185-191.

3. Hadany $L$, Comeron JM: Why are sex and recombination so common? Ann N Y Acad Sci 2008, 1133:26-43.

4. Casselton LA: Mate recognition in fungi. Heredity 2002, 88:142-147.

5. Fraser JA, Heitman J: Evolution of fungal sex chromosomes. Mol Microbiol 2004, 51:299-306.

6. Lee SC, Ni M, Li W, Shertz C, Heitman J: The evolution of sex: a perspective from the fungal kingdom. Microbiol Mol Biol Rev 2010, 74:298-340.

7. Billiard S, López-Villavicencio M, Devier B, Hood ME, Fairhead C, Giraud T: Having sex, yes, but with whom? Inferences from fungi on the evolution of anisogamy and mating types. Biol Rev 2011, 86:421-442.

8. Fraser JA, Heitman J: Chromosomal sex-determining regions in animals, plants and fungi. Curr Opin Genet Dev 2005, 15:645-651.

9. Fraser JA, Heitman J: Fungal mating-type loci. Curr Biol 2003, 13:R792-795.

10. Gryganskyi AP, Lee SC, Litvintseva AP, Smith ME, Bonito G, Porter TM, Anishchenko IM, Heitman J, Vilgalys R: Structure, function, and phylogeny of the mating locus in the Rhizopus oryzae complex. PLoS One 2010, 5: e15273.

11. Idnurm A, Walton FJ, Floyd A, Heitman J: Identification of the sex genes in an early diverged fungus. Nature 2008, 451:193-196.

12. Lee SC, Corradi N, Byrnes EJ, Torres-Martinez S, Dietrich FS, Keeling PJ, Heitman J: Microsporidia evolved from ancestral sexual fungi. Curr Biol 2008, 18:1675-1679.

13. Fraser JA, Hsueh YP, Findley KM, Heitman J: Evolution of the mating-type locus: the basidiomycetes. In Sex in fungi: Molecular determination and evolutionary implications. Edited by: Heitman J, Kronstad JW, Taylor JW Casselton LA. Washington D.C.: ASM Press; 2007:19-34.

14. Raudaskoski $\mathrm{M}$, Kothe $\mathrm{E}:$ Basidiomycete mating type genes and pheromone signaling. Eukaryot Cell 2010, 9:847-859.

15. Xue C, Hsueh YP, Heitman J: Magnificent seven: roles of G proteincoupled receptors in extracellular sensing in fungi. FEMS Microbiol Rev 2008, 32:1010-1032.

16. Bakkeren G, Kronstad JW: Linkage of mating-type loci distinguishes bipolar from tetrapolar mating in basidiomycetous smut fungi. Proc Natl Acad Sci USA 1994, 91:7085-7089.

17. Kües U, Richardson WV, Tymon AM, Mutasa ES, Göttgens B, Gaubatz $S$, Gregoriades A, Casselton LA: The combination of dissimilar alleles of the $A a$ and $A \beta$ gene complexes, whose proteins contain homeo domain motifs, determines sexual development in the mushroom Coprinus cinereus. Genes Dev 1992, 6:568-577.

18. Banham AH, Asante-Owusu RN, Göttgens B, Thompson S, Kingsnorth CS, Mellor $\mathrm{E}$, Casselton LA: An N-terminal dimerization domain permits homeodomain proteins to choose compatible partners and Initiate sexual development in the mushroom Coprinus cinereus. Plant Cell 1995, 7:773-783.
19. Kämper J, Reichmann M, Romeis T, Bölker M, Kahmann R: Multiallelic recognition: nonself-dependent dimerization of the $\mathrm{bE}$ and bW homeodomain proteins in Ustilago maydis. Cell 1995, 81:73-83.

20. Casselton $L A$, Kües U: The origin of multiple mating types in the model mushrooms Coprinopsis cinerea and Schizophyllum commune. In Sex in fungi: Molecular determination and evolutionary implications. Edited by: Heitman J, Kronstad JW, Taylor JW, Casselton LA. Washington D.C.: ASM Press; 2007:283-300.

21. Casselton LA, Olesnicky NS: Molecular genetics of mating recognition in basidiomycete fungi. Microbiol Mol Biol Rev 1998, 62:55-70.

22. Kothe E: Tetrapolar fungal mating types: sexes by the thousands. FEMS Microbiol Rev 1996, 18:65-87.

23. Raper JR: Genetics of sexuality in Higher Fungi New York: The Roland Press; 1966.

24. Coelho MA, Sampaio JP, Gonçalves P: A deviation from the bipolartetrapolar mating paradigm in an early diverged basidiomycete. PLOS Genet 2010, 6:e1001052.

25. Stajich JE, Berbee ML, Blackwell M, Hibbett DS, James TY, Spatafora JW, Taylor JW: The fungi. Curr Biol 2009, 19:R840-845.

26. Lin X, Heitman J: Mechanisms of homothallism in Fungi and transitions between heterothallism and homothallism. In Sex in fungi: Molecular determination and evolutionary implications. Edited by: Heitman J, Kronstad JW, Taylor JW, Casselton LA. Washington D.C.: ASM Press; 2007:35-57.

27. Kondrashov AS: Classification of hypotheses on the advantage of amphimixis. J Hered 1993, 84:372-387.

28. Maynard-Smith J: The Evolution of Sex. 1 edition. Cambridge, UK: Cambridge University Press; 1978.

29. Bell G: The masterpiece of nature: the evolution and genetics of sexuality London, United Kingdom: CroomHelm; 1982

30. Aanen DK, Hoekstra RF: Why sex is good: on fungi and beyond. In Sex in fungi: Molecular determination and evolutionary implications. Edited by: Heitman J, Kronstad JW, Taylor JW, Casselton LA. Washington D.C.: ASM Press; 2007:527-534.

31. Begerow D, Bauer R, Boekhout T: Phylogenetic placements of ustilaginomycetous anamorphs as deduced from nuclear LSU rDNA sequences. Mycol Res 2000, 104:53-60

32. Kurtzman $\mathrm{CP}$, Robnett $\mathrm{CJ}$ : Identification and phylogeny of ascomycetous yeasts from analysis of nuclear large subunit (26S) ribosomal DNA partial sequences. Antonie Van Leeuwenhoek 1998, 73:331-371.

33. Hibbett DS, Binder M, Bischoff JF, Blackwell M, Cannon PF, Eriksson OE, Huhndorf S, James T, Kirk PM, Lucking R, et al: A higher-level phylogenetic classification of the Fungi. Mycol Res 2007, 111:509-547.

34. Hawksworth DL: Naming Aspergillus species: progress towards one name for each species. Med Mycol 2011, 49(Suppl 1):S70-76.

35. Sampaio JP, Gadanho M, Bauer R, Weiss M: Taxonomic studies in the Microbotryomycetidae Leucosporidium golubevii sp. nov., Leucosporidiella gen. nov. and the new orders Leucosporidiales and Sporidiobolales. Mycol Prog 2003, 2:53-68

36. Sampaio JP: Rhodosporidium Banno. In The Yeasts, a taxonomic study, Volume 3, Part Vb. 5 edition. Edited by: Kurtzman CP, Fell JW, Boekhout T. Amsterdam, The Netherlands: Elsevier; 2011:1523-1540

37. Devier B, Aguileta G, Hood ME, Giraud T: Ancient trans-specific polymorphism at pheromone receptor genes in basidiomycetes. Genetics 2009, 181:209-223.

38. Muirhead CA, Glass NL, Slatkin M: Multilocus self-recognition systems in fungi as a cause of trans-species polymorphism. Genetics 2002, 161:633-641.

39. Coelho MA, Rosa A, Rodrigues N, Fonseca Á, Gonçalves P: Identification of mating type genes in the bipolar basidiomycetous yeast Rhodosporidium toruloides: first insight into the MAT locus structure of the Sporidiobolales. Eukaryot Cell 2008, 7:1053-1061.

40. Sampaio JP: Rhodotorula Harrison. In The Yeasts, a taxonomic study, Volume 3, Part VC. 5 edition. Edited by: Kurtzman CP, Fell JW, Boekhout T. Amsterdam, The Netherlands: Elsevier; 2011:1873-1928.

41. Gadanho M, Sampaio JP: Polyphasic taxonomy of the basidiomycetous yeast genus Rhodotorula Rh. glutinis sensu stricto and Rh. dairenensis comb. nov. FEMS Yeast Res 2002, 2:47-58.

42. Taylor J, Jacobson D, Fisher M: The evolution of asexual fungi: reproduction, speciation and classification. Annu Rev Phytopathol 1999, 37:197-246. 
43. Hull CM, Heitman J: Genetics of Cryptococcus neoformans. Annu Rev Genet 2002, 36:557-615.

44. Lin X, Litvintseva AP, Nielsen $\mathrm{K}$, Patel S, Floyd A, Mitchell TG, Heitman J: aADa hybrids of Cryptococcus neoformans: evidence of same-sex mating in nature and hybrid fitness. PLoS Genet 2007, 3:1975-1990.

45. López-Villavicencio M, Aguileta G, Giraud T, de Vienne DM, Lacoste S, Couloux A, Dupont J: Sex in Penicillium: combined phylogenetic and experimental approaches. Fungal Genet Biol 2010, 47:693-706.

46. O'Donnell K, Ward TJ, Geiser DM, Corby Kistler H, Aoki T: Genealogical concordance between the mating type locus and seven other nuclear genes supports formal recognition of nine phylogenetically distinct species within the Fusarium graminearum clade. Fungal Genet Biol 2004, 41:600-623.

47. Banuett F, Herskowitz I: Different $a$ alleles of Ustilago maydis are necessary for maintenance of filamentous growth but not for meiosis. Proc Natl Acad Sci USA 1989, 86:5878-5882.

48. Hull CM, Boily MJ, Heitman J: Sex-specific homeodomain proteins Sxi1a and Sxi2a coordinately regulate sexual development in Cryptococcus neoformans. Eukaryot Cell 2005, 4:526-535.

49. Hull CM, Davidson RC, Heitman J: Cell identity and sexual development in Cryptococcus neoformans are controlled by the mating-type-specific homeodomain protein Sxi1a. Genes Dev 2002, 16:3046-3060.

50. Fowler TJ, Mitton MF, Vaillancourt LJ, Raper CA: Changes in mate recognition through alterations of pheromones and receptors in the multisexual mushroom fungus Schizophyllum commune. Genetics 2001, 158:1491-1503.

51. Olesnicky NS, Brown AJ, Dowell SJ, Casselton LA: A constitutively active Gprotein-coupled receptor causes mating self-compatibility in the mushroom Coprinus. EMBO J 1999, 18:2756-2763.

52. Olesnicky NS, Brown AJ, Honda Y, Dyos SL, Dowell SJ, Casselton LA: Selfcompatible $B$ mutants in Coprinus with altered pheromone-receptor specificities. Genetics 2000, 156:1025-1033

53. Devier B, Aguileta G, Hood ME, Giraud T: Using phylogenies of pheromone receptor genes in the Microbotryum violaceum species complex to investigate possible speciation by hybridization. Mycologia 2010, 102:689-696.

54. Fraser JA, Diezmann S, Subaran RL, Allen A, Lengeler KB, Dietrich FS, Heitman J: Convergent evolution of chromosomal sex-determining regions in the animal and fungal kingdoms. PLoS Biol 2004, 2:e384.

55. Chen JM, Cooper DN, Chuzhanova N, Férec C, Patrinos GP: Gene conversion: mechanisms, evolution and human disease. Nat Rev Genet 2007, 8:762-775

56. Rozen S, Skaletsky H, Marszalek JD, Minx PJ, Cordum HS, Waterston RH, Wilson RK, Page DC: Abundant gene conversion between arms of palindromes in human and ape $\mathrm{Y}$ chromosomes. Nature 2003, 423:873-876

57. Charlesworth D, Charlesworth B, Marais G: Steps in the evolution of heteromorphic sex chromosomes. Heredity 2005, 95:118-128.

58. Yang Z: PAML 4: Phylogenetic Analysis by Maximum Likelihood. Mol Biol Evol 2007, 24:1586-1591.

59. Higgs PG, Ran W: Coevolution of codon usage and tRNA genes leads to alternative stable states of biased codon usage. Mol Biol Evol 2008, 25:2279-2291.

60. Clark AG, Kao TH: Excess nonsynonymous substitution of shared polymorphic sites among self-incompatibility alleles of Solanaceae. Proc Natl Acad Sci USA 1991, 88:9823-9827.

61. Hinata $K$, Watanabe $M$, Yamakawa S, Satta $Y$, Isogai A: Evolutionary aspects of the S-related genes of the Brassica self-incompatibility system: synonymous and nonsynonymous base substitutions. Genetics 1995, 140:1099-1104

62. Emes RD, Yang Z: Duplicated paralogous genes subject to positive selection in the genome of Trypanosoma brucei. PLoS One 2008, 3:e2295

63. Whittle CA, Nygren K, Johannesson H: Consequences of reproductive mode on genome evolution in fungi. Fungal Genet Biol 2011, 8:661-7.

64. Whittle CA, Sun Y, Johannesson H: Degeneration in codon usage within the region of suppressed recombination in the mating type chromosomes of Neurospora tetrasperma. Eukaryot Cell 2011, 10:594-603.

65. Woo PC, Chong KT, Tse H, Cai JJ, Lau CC, Zhou AC, Lau SK, Yuen KY: Genomic and experimental evidence for a potential sexual cycle in the pathogenic thermal dimorphic fungus Penicillium marneffei. FEBS Lett 2006, 580:3409-3416
66. Muller HJ: Some Genetic Aspects of Sex. Am Nat 1932, 66:118-138

67. Otto SP, Lenormand T: Resolving the paradox of sex and recombination. Nat Rev Genet 2002, 3:252-261.

68. Thompson JD, Higgins DG, Gibson TJ: CLUSTAL W: improving the sensitivity of progressive multiple sequence alignment through sequence weighting, position-specific gap penalties and weight matrix choice. Nucleic Acids Res 1994, 22:4673-4680.

69. Hall TA: BioEdit: a user-friendly biological sequence alignment editor and analysis program for Windows 95/98/NT. Nucl Acids Symp Ser 1999, 41:95-98.

70. Guindon S, Gascuel O: A simple, fast, and accurate algorithm to estimate large phylogenies by maximum likelihood. Syst Biol 2003, 52:696-704.

71. Posada D, Crandall KA: MODELTEST: testing the model of DNA substitution. Bioinformatics 1998, 14:817-818.

72. Edgar RC: MUSCLE: multiple sequence alignment with high accuracy and high throughput. Nucleic Acids Res 2004, 32:1792-1797.

73. Stanke M, Diekhans M, Baertsch R, Haussler D: Using native and syntenically mapped CDNA alignments to improve de novo gene finding. Bioinformatics 2008, 24:637-644.

74. Abascal F, Zardoya R, Posada D: ProtTest: selection of best-fit models of protein evolution. Bioinformatics 2005, 21:2104-2105.

75. Adachi J, Hasegawa M: Model of amino acid substitution in proteins encoded by mitochondrial DNA. J Mol Evol 1996, 42:459-468.

76. Cao Y, Adachi J, Janke A, Pääbo S, Hasegawa M: Phylogenetic relationships among eutherian orders estimated from inferred sequences of mitochondrial proteins: instability of a tree based on a single gene. $J$ Mol Evol 1994, 39:519-527.

77. Yang Z: Maximum-likelihood estimation of phylogeny from DNA sequences when substitution rates differ over sites. Mol Biol Evol 1993, 10:1396-1401.

78. Whelan S, Goldman N: A general empirical model of protein evolution derived from multiple protein families using a maximum-likelihood approach. Mol Biol Evol 2001, 18:691-699.

79. Nielsen R, Yang Z: Likelihood models for detecting positively selected amino acid sites and applications to the HIV-1 envelope gene. Genetics 1998, 148:929-936.

80. Swanson WJ, Nielsen R, Yang Q: Pervasive adaptive evolution in mammalian fertilization proteins. Mol Biol Evol 2003, 20:18-20.

81. Yang Z, Nielsen R, Goldman N, Pedersen A-MK: Codon-substitution models for heterogeneous selection pressure at amino acid sites. Genetics 2000, 155:431-449.

82. Wong WSW, Yang Z, Goldman N, Nielsen R: Accuracy and power of statistical methods for detecting adaptive evolution in protein coding sequences and for identifying positively selected sites. Genetics 2004, 168:1041-1051

83. Yang Z, Wong WS, Nielsen R: Bayes empirical bayes inference of amino acid sites under positive selection. Mol Biol Evol 2005, 22:1107-1118.

doi:10.1186/1471-2148-11-249

Cite this article as: Coelho et al: Evidence for maintenance of sex determinants but not of sexual stages in red yeasts, a group of early diverged basidiomycetes. BMC Evolutionary Biology 2011 11:249.

\section{Submit your next manuscript to BioMed Central and take full advantage of:}

- Convenient online submission

- Thorough peer review

- No space constraints or color figure charges

- Immediate publication on acceptance

- Inclusion in PubMed, CAS, Scopus and Google Scholar

- Research which is freely available for redistribution 